\title{
Co-evolution Mechanism and Stability Analysis of Service-oriented Manufacturing Enterprise System
}

\author{
Peipei Liang ${ }^{1,2}$,Yanming Sun ${ }^{1}$, Yali Pan ${ }^{3 *}$ \\ ${ }^{1}$ School of Business Administration, South China University of Technology (China) \\ ${ }^{2}$ Geological Resource Management Research Center, East China Institute of Technology (China) \\ ${ }^{3}$ School of Mathematical Science, Huaibei Normal University (China) \\ 614lpp@163.com, meymsun@scut.edu.cn,*Corresponding author:panliiziang@163.com
}

Received: April 2015

Accepted: October 2015

\section{Abstract:}

Purpose: Service-oriented manufacturing (SOM) is a new worldwide trend in the manufacturing industry. An increasing number of enterprises have realized the advantages and importance of SOM. However, the co-evolution mechanism and the stability of serviceoriented manufacturing enterprise systems have influences on the form and direction of evolution and decisions about how to determine the suitable scope of external investment. This paper aims to reveal the co-evolution mechanism and provide a scientific framework for the efficient operation of SOM enterprise system.

Design/methodology/approach: Based on a system's features and the logistic equation, our research builds a three-dimensional dynamic model with three state variables: response capability, profitability, and structural complexity. In addition, an analysis of a system's stability and state variables is conducted using the method of synergetics and, simultaneously, the threshold condition of co-evolution was determined and the evolution mechanism revealed. Finally, model validity is verified through the simulation of instance.

Findings: By analyzing the dynamic model using Lyapounov stability analysis, the results show that responsiveness under the threshold condition will guide an enterprise system's evolution for a long period of time, and whether the SOM enterprise system is stable or not depends on 
the external investment level $\theta$. When the investment level reaches the threshold condition, system will realize its development and then repeatedly change from one kind of structure to another for an extended period. If not, system will be unstable, although it will improve, the rise in responsiveness will not be obvious.

Originality/value: Most of the related researches are over-reliant on qualitative description and has seldom been from the perspective of a complex system. This work is a supplement from evolution perspective to SOM related theory researches, and also an innovation in the context of complexity science methods applied in enterprise management field

Keywords: service-oriented manufacturing, complex system, dynamic model co-evolution, stability

\section{Introduction}

In recent years, some obvious environmental and industrial changes (e.g., shifts in consumer behavior, transformations of enterprise operation patterns and trends in networked enterprise services) have made the servitization of manufacturing a worldwide trend. Since the late 1980s, servitization has gradually become integrated with the traditional manufacturing industry, and this has resulted in the development of an advanced manufacturing mode, known as serviceoriented manufacturing (SOM) (Fry, Steele \& Saladin, 1994). In the 20th century, some enterprises engaged in vertical integration to control their supply chains, and then went a step further to bundle goods and services together for customers. Some well-known companies, including GE and IBM, took the lead in transitioning from goods to services, which made them strongly competitive. In the past few decades, these companies have maintained growth rates in excess of $10 \%$, with more than $50 \%$ of their revenue coming from service provision.

Traditionally, manufacturing companies have focused their efforts on designing, developing and producing physical products to offer on the market. Recently, manufacturing industries face a strong trend towards servitization. With the shift from a product-orientation to service-orientation, they pay more attention to the integration of products and services. In a service perspective, value is derived from customers' satisfaction with the services and the use of products, which also brings new challenges for manufacturing enterprises. These may need a large amount of resources to understand customers' requirements, collaborate with upstream and downstream enterprises, and then respond rapidly to the market; nevertheless, the effect of input-output is always not obvious. Hence it is necessary to study the impact of input level on the evolution mechanism of enterprise systems.

This paper focuses on the evolution rule and evolution stability analysis of an SOM enterprise system, and we present it in the following format: in section 2 , an overview of the relevant 
researches is presented. In section 3, following the selection of state variables and order parameter, a co-evolution model is formed based on a logistic equation. Following that, section 4 provides an analysis of evolution stability, referring to synergetics and establishes a threshold condition in a system's development. Then, in section 5, a simulation example is provided and its results analyzed. Finally, concluding remarks and comments relating to future research are provided in section 6.

\section{Literature Review}

Our work is to analyze the co-evolution mechanism from the perspective of complex system and provide a scientific framework for the efficient operation of SOM enterprise system. As such, the overview of the most relevant literatures is presented from two aspects. The first is related to the SOM research status, and the second is related to the complexity science method.

The roots of SOM can be traced back to the rise of the concept of "producer services" (Greenfield, 1996), which focuses on how to manufacture products based on outsourcing services from other suppliers, but is not concerned with the target of the services. Previous studies have been performed to verify the positive influence on the national economy of developing the "producer services" industry, and to investigate the micro mechanisms of producer services (Macpherson, 1997; Gunasekaran \& Spalanzani, 2012). Several researchers believe that the key to establishing competitiveness is to create value by understanding customers' requirements, involving the participation of customers in the entire process of manufacturing, and affording them a set of tangible and customized services (Grönroos, 2000; Schmenner, 2004, 2009).

Since the birth of the SOM concept, there has been a steady flow of studies and programmers (Lightfoot, Baines \& Smart, 2013). Nariaki and Wang (2013) proposed five kinds of game theories for platform-type product service systems considering manufacturing viewpoints. They mainly focused on the framework incorporating products and services for the solution to servicization. Mendes, Leitao, Colombo and Restivo (2012) introduced an integrated approach for the design, analysis, validation, simulation, and process execution of SOM systems by using high-level petri nets. Zhen (2012) performed (and presented) an analytical study of the objectives of an optimal decision model for designing an SOM strategy. Huang, Zeng, Fan and Huang (2011) pointed out that service management is the core content of SOM and proposed a comprehensive performance evaluation metric for SOM networks. Franco, Bas and Esteban (2009) designed an architectural style of SOM platform, and $\mathrm{Wu}, \mathrm{Xi}$ and Zhou (2008) presented manufacturing communication architecture based on messaging and web services. For those papers mentioned, most papers discussing SOM focus on concept, architecture, 
strategies, implementation methods, appreciation, and value-adding mechanisms. The study of SOM in term of evolution mechanisms remains weak from the perspective of complex systems.

Enterprise systems have already been shown to be complex systems with the characteristics of openness, non-linear interaction, non-equilibrium state, and large fluctuations (Basole \& Bodner, 2015). As a result of a subsystem's self-organization and co-evolution, a SOM enterprise system, also a complex system, has been shown to have a relatively stable state from the macro perspective. However, the micro units are not stable, especially when an enterprise's operation and development is under the influence of the ever-changing market environment. Several theories have provided us with novel research methods for studying the evolution of complex systems, such as multi-agent theory (Paulo, Jose \& Damien, 2012), bionics theory (Tharumarajah, 2003), fractal theory (Shin, Mun \& Jung, 2009), and dynamic theory (Sarimveis, Patrinos, Tarantilis \& Kiranoudis, 2008). Those show that complexity science methods are appropriate for the research questions, and have contributed a lot to this area. In addition, synergetic theory, the main research method of complex systems, has two important basic principles: Haken's slaving principle and order parameter (Tschacher \& Haken, 2007). The slaving principle reveals how the upper layer dominates the layer below in the transition from a disordered state to an ordered state in a complex system. As a rare parameter at the top of a system, the order parameter, despite its low speed, controls the entire macroscopic system in its evolution from a disordered state to an ordered state. Our paper uses synergetic theory to investigate the evolution mechanism and stability, while most papers focus on building conceptual frameworks or performing some empirical studies. Moreover, few scholars had studied the impact of input level on the form and direction of evolution, this study has a research on the threshold condition of input level to guide system evolution.

\section{Construction of Co-Evolution Model}

\subsection{Selection of the State Variables and Order Parameter}

The state variables and the control variable are two important factors in defining a system. The state variables represent the performance state in a system's evolution process, while the control variable is a controllable factor and a condition variable that allows a system to achieve threshold value and co-evolution. According to Haken's slaving principle, the first step is to immediately confirm the order parameter that relates to each system. Although there are more state variables during the evolution process, only a few major state variables have a significant effect on a system's evolution. The guiding and dominating function of the order parameter in the process of a system's development directly affects the direction and initial results of a system's evolution. As such, it is necessary to simply and scientifically describe a complicated system and find the determining and controlling factors in its evolution. An SOM system can be a single manufacturing enterprise or a cooperation alliance composed of mutually 
advantageous enterprises in the manufacturing supply chain. Both are able to respond quickly to market opportunities. The former is the research objective of this paper; the latter will be studied at a later date.

The order parameter relates to the state variables of an enterprise system that are produced internally, in addition to the main characteristics that have a long-term effect on an enterprise system's operation. Increasingly, with the ever-changing market environment, responding rapidly to market cues is the key to success. When the control variable reaches the threshold condition, the variable of responsiveness will guide an enterprise system's evolution for a long period of time. As such, responsiveness under the threshold condition is a characteristic of the order parameter, and so we regard the target responsibility that responds rapidly to the manufacturing system as the primary macro order parameter of a system. The ultimate aim of a system is to raise its responsiveness to gain greater profits and a larger market share. Consequently, we have used the state variable, profitability, to describe the business state of a system. At the same time, to rapidly respond to changing market demands, the structure of an SOM enterprise system must transform to become distributed, self-managing and a tabular net structure, instead of remaining static. We have chosen structural complexity as a state variable to reflect the state of a system's resource and the state of their information flow for daily decisions relating to a system's responsiveness.

\subsection{The Dynamic Model of System Co-evolution}

Our research aims to reveal a system's evolution mechanism by forming a dynamic model of co-evolution and establishing the condition by analyzing the stability and state variables of each system. Apart from complicated self-organization conditions such as system opening, non-equilibrium state, non-linear interaction, and system fluctuations (Qu \& Ortoleva, 2007); an SOM enterprise system also has to meet the following assumed conditions.

1. A system's development must be confined to elements like manpower, capital, and technology. Moreover, it must set administrative decisions (regarded as investment decisions) as the control variable in the model.

2. The conditions for system instability may be various, as there is a one-one correlation between the threshold condition and the critical value of instability. Moreover, during its evolution, a change in a system's control variable will lead to a change in the system's instability condition.

3. A system's development must have mathematical continuity. Relative parameters in the model can be quantified by a specific index. 
Before forming a specific model, we use $q_{1}, q_{2}, q_{3}$ to represent a system's responsiveness, profitability and structural complexity, respectively. $\alpha, \beta, \gamma$, respectively, are used as adjustment factors for the state variables $q_{1}, q_{2}, q_{3}$ (factors that interact with each other and statistics that can be measured by an index system), and $\theta$ is used to represent the control variable, which is regarded as a system investment composite index. Because of these assumptions, the control variable of the three state variables is always $\theta$. A manufacturing enterprise system's responsiveness, $q_{1}$, is related to structural complexity, $q_{3}$. In general, a system's responsiveness to market demands will gradually decrease, while its degree of structural complexity becomes greater at the early stage of system evolution. However, whether or not a system is making a profit has no influence on a company's responsiveness to market demands. Under the condition of system investment, $\theta$, the logistic evolution of state variable $q_{1}$ shows:

$$
\frac{1}{\alpha} \frac{d q_{1}}{d t}=\theta q_{1}-\theta \frac{\gamma}{\alpha} q_{3}-\gamma q_{1} q_{3}+\varphi_{1} q_{3}^{k}
$$

$\theta q_{1}$ represents the self-feedback function of $q_{1}$ under the effect of $\theta$. $-\theta \gamma q_{3} / \alpha$ describes the negative correlation between structural complexity and responsiveness affected by control variable $\theta$, and $\gamma / \alpha$ represents the impact factor; $-\gamma q_{1} q_{3}$ means a system's responsiveness reduces, while the structural complexity expands; $\varphi_{1} q_{3}{ }^{k}$ represents the relevant information between structural complexity and profitability, with $\varphi_{1}, k$ as constants. A systematic and rational management structure always brings greater benefit to a system. We therefore presume $\varphi_{1}=2, k=2$ to represent a system's present benefit and the effect of increased structure.

With regards to the profitability, $q_{2}$, of the manufacturing enterprise system, its evolution is only related to its self-state and system responsiveness, and has no direct relationship with a system's structural complexity. For instance, the profitability of large enterprises with a complicated structure is not always higher than that of small enterprises with a simple structure or medium-sized ones with a moderately complex structure. Hence, the logistic evolution of state variable $q_{2}$ can be represented as follows:

$$
\frac{1}{\beta} \frac{d q_{2}}{d t}=-\varphi_{2} q_{2}+\varphi_{3} \theta \frac{\alpha}{\beta} q_{1}
$$

$-\varphi_{2} q_{2}$ represents the relationship between $q_{2}$ and its own state. Without the effect of the control variable, $q_{2}$ tends to decline, as it has no direct relationship with $\theta . \varphi_{2}$ is constant, assuming that $\varphi_{2}=1$ means that the enterprise's profitability can be maintained without any outside influence; $\theta \alpha \varphi_{3} \theta_{1} / \beta$ means that a system's profitability can be improved by increasing responsiveness; $\alpha / \beta$ is the impact factor; and $\varphi_{3}$ is constant, assuming that $\varphi_{3}=2$ represents that a system has a strong learning ability. 
For a system's structural complexity, $q_{3}$, the initial cell structure is the basis of the enterprise's responsiveness and profitability. Thus, $q_{3}$ is closely linked with a system's responsiveness and profitability. In addition, a system's continuous development is based on its present structure, so $q_{3}$ is related to itself. Hence, the logistic evolution of state variable $q_{3}$ can be expressed as follows:

$$
\frac{1}{\gamma} \frac{d q_{3}}{d t}=-\theta q_{3}+\alpha q_{1} q_{3}+\frac{\beta}{\gamma} q_{2}
$$

$-\theta q_{3}$ relates to the self-feedback function of $q_{3}$ under the effect of $\theta$. A negative coefficient means marginal structural complexity and a progressively decreasing benefit. The description of a system's development is restrained. The profitability will be restricted when imprudent investments result in structural complexity surpassing a system's threshold value. $\alpha q_{1} q_{3}$ represents the positive effect of system responsiveness on structural complexity. $\beta q_{2} / \gamma$ means the promoting role of enterprise benefits its structure. $\beta / \gamma$ is the impact factor.

Summarizing and simplifying the above, we form the idea of a co-evolution model of an SOM enterprise system as below:

$$
\left\{\begin{array}{c}
\frac{d q_{1}}{d t}=\alpha \theta q_{1}-\theta \gamma q_{3}-\alpha \gamma q_{1} q_{3}+2 \alpha q_{3}^{2} \\
\frac{d q_{2}}{d t}=-\beta q_{2}+2 \theta \alpha q_{1} \\
\frac{d q_{3}}{d t}=-\theta \gamma q_{3}+\alpha \gamma q_{1} q_{3}+\beta q_{2}
\end{array}\right.
$$

Definitions of $\theta, \alpha, \beta, \gamma$ respectively, as follows:

$\theta=\sqrt[3]{\prod_{i=1}^{3} \frac{\theta_{i}}{\bar{\theta}_{l}} k_{\theta i}}$ represents the comprehensive index of system investment. $\theta_{i}(i=1,2,3)$ represents, respectively, the capitalizing investment of manpower, capital, and technology, as well as forming the criterion for every index of the benchmarking system. $K_{\theta}$ is the index weight coefficient.

$\alpha=\sqrt[3]{\frac{\overline{\alpha_{1}}}{\alpha_{1}} k_{a 1} \prod_{i=2}^{3} \frac{\alpha_{i}}{\frac{\alpha_{1}}{\alpha_{a i}}}}$ represents the level index of system responsiveness. $\alpha_{i}(i=1,2,3)$ represents, respectively, order lead time, fill rate, and species of new products, as well as the criterion for every index of the benchmarking system. $K_{a i}$ is the index weight coefficient.

$\beta=\sqrt{\prod_{i=1}^{2} \frac{\beta_{i}}{\bar{\beta}_{l}} k_{\beta i}}$ represents the level index of system profitability. $\beta_{i}(i=1,2)$ represents, respectively, the profit rate and market share, as well as the criterion for every index of the benchmarking system. $K_{\beta i}$ is the index weight coefficient. 
$\gamma=\sqrt[3]{\prod_{i=1}^{3} \frac{\gamma_{i}}{\bar{\gamma}_{\iota}} k_{\gamma i}}$ represents the index of a system's structural complexity, described as the structural entropy. $\gamma_{i}(i=1,2,3)$ is chosen to measure system complexity with management span entropy, decision path entropy and information communication entropy. $\overline{\gamma_{l}}$ represents the criterion for every index of the benchmarking system. $K_{\gamma i}$ is the index weight coefficient. Where $\gamma_{i}=1-S_{i} / S_{m i}, S_{i}=-\sum_{i=1}^{n_{i}} p_{i} \log _{2} p_{i}, p_{i}$ represents the microscopic state probability; $S_{m i}$ is the largest entropy; and $n_{i}$ means the total number of a system's microscopic states. If $S_{m i}=\max \left(-\sum_{i=1}^{n_{i}} p_{i} \log _{2} p_{i}\right)$, this can be achieved by solving the equation using Lagrange, regarding $p_{i}$ as an independent vector, working out the partial derivatives and obtaining $\left.S_{m i}=\log _{2} n_{i}\right)$.

\section{Model Analysis}

\subsection{Lyapounov Stability Analysis}

The purpose of analyzing a system's stability is on the one hand to discover a situation in which a system's original reference state may have lost its stability, and on the other hand to set the threshold value of a system's co-development, because a system's development is to realize the servo to order parameter by constantly exceeding the limits of the threshold value. We will use Lyapounov's indirect method to distinguish system stability. Lyapounov's indirect method relates to non-linear systems and linearizing a non-linear state equation around an equilibrium state, then solving eigenvalue of the linear state equation set, and determining the system stability according to the distribution of all of the eigenvalues in a complex plane.

If we let $d q_{i} / d t=0$, because it is simple to verify that $(0,0,0)$ is the steady state solution of evolution Equation (1), then we can achieve the following equations by linearization.

$$
\left\{\begin{array}{l}
\frac{d q_{1}}{d t}=\alpha \theta q_{1}-\theta \gamma q_{3} \\
\frac{d q_{2}}{d t}=-\beta q_{2}+2 \theta \alpha q_{1} \\
\frac{d q_{3}}{d t}=-\theta \gamma q_{3}+\beta q_{2}
\end{array}\right.
$$

Written in matrix form: $d q / d t=A q$, where $A=\left[\begin{array}{ccc}\alpha \theta & 0 & -\theta \gamma \\ 2 \alpha \theta & -\beta & 0 \\ 0 & \beta & -\theta \gamma\end{array}\right]$. According to the eigenvalue equation $|A-\lambda E|=0$, we obtain: 


$$
\begin{gathered}
\left|\begin{array}{ccc}
\alpha \theta-\lambda & 0 & -\theta \gamma \\
2 \alpha \theta & -\beta-\lambda & 0 \\
0 & \beta & -\theta \gamma-\lambda
\end{array}\right|=0 \\
\Rightarrow \lambda^{3}+(\theta \gamma+\beta-\alpha \theta) \lambda^{2}+\left(\theta \beta \gamma-\alpha \gamma \theta^{2}-\alpha \beta \theta\right) \lambda+\alpha \beta \gamma \theta^{2}=0
\end{gathered}
$$

According to Lyapounov's indirect method, all eigenvalues of Matrix A have a negative real part. Hence, the stability of a non-linear system becomes asymptotically unstable. A system will likely reach the threshold only under the condition of instability. Based on Routh-Hurwitz's Criterion, we can achieve the following conditions for a system's instability.

$$
\left\{\begin{array}{l}
\theta \gamma+\beta-\alpha \theta>0 \\
\alpha \beta \gamma \theta^{2}>0 \\
(\theta \gamma+\beta-\alpha \theta)\left(\theta \beta \gamma-\alpha \gamma \theta^{2}-\alpha \beta \theta\right)-\alpha \beta \gamma \theta^{2}>0
\end{array}\right.
$$

Letting the right-hand side of Equation (5) equal zero; we can obtain two solutions for the control variable $\theta$, which are, respectively, $\beta /(\alpha-\gamma), \beta(\gamma-\alpha) / \alpha \gamma$.

(i) If $\alpha=\gamma$, no matter what the value of control variable $\theta$, conditions (3),(4) are always established, but condition (5) is always less than zero.

(ii) If $\alpha<\gamma$, we can achieve the condition for a system's instability by solving inequalities (3), (4), (5), which is $\beta /(\alpha-\gamma)<\theta<\beta(\gamma-\alpha) / \alpha \gamma$. The further simplification is: $0<\theta<\beta(\gamma-\alpha) / \alpha \gamma$

(iii) If $\alpha>\gamma, \theta<\beta(\gamma-\alpha) / \alpha \gamma<0$ can be obtained by solving inequalities (3), (4), (5), then $\theta<0$ has no practical meaning.

Equally, if $\alpha \geq \gamma$, then a system's equilibrium state is stable and has no bearing on control variable $\theta_{\text {; }}$ if $\alpha<\gamma$, then the condition for a system's instability is $0<\theta<\beta(\gamma-\alpha) / \alpha \gamma$ and the threshold condition of the system is $\theta=\beta(\gamma-\alpha) / \alpha \gamma$.

Because $\alpha$ and $\gamma$ are compared to professional standards, (i) $\alpha \geq \gamma$ means that the responsiveness of systems with equal structural complexity will be more than or equal to that of the benchmarking manufacturing enterprise system. Upon taking a leading position in the industry, a system's strategy (including the adjustment of the system's structure, marketing strategy and investment decisions) will not easily change in the short term. Therefore, a system will be stable under these conditions, which matches the situation in reality. (ii) $\alpha<\gamma$ means that the responsiveness of a system with equal structural complexity will be lower than that of the benchmarking manufacturing enterprise system. Only when the investment level reaches $\beta(\gamma-\alpha) / \alpha \gamma$, the system will break the threshold value and realize its development. It will then repeatedly change from one kind of structure to another for an extended period. 
When the investment level is in the region of $[0, \beta(\gamma-\alpha) / \alpha \gamma)$ and does not meet the requirements of the threshold value, the system's responsiveness will be unstable, and, although it will improve, the rise in responsiveness will not be obvious, as responsiveness is related to the investment level. Only breaking the threshold value will bring significant improvements to a system. A system's investments are influenced by external and internal factors (such as market, policy, and strategy), and unlimited investment will ultimately bring greater risk. The misapprehension that unlimited investment can continuously improve a system's responsiveness should be avoided.

\subsection{Evolutionary Analysis of State Variables}

As the key method used to simplify problems in synergetics, Haken's adiabatic approximation is the primary means of looking for the order parameter. It can also indicate a system's co-evolution rule under the effect of the order parameter. The changing speed of each state variable in a system's process of evolution can be found intuitively. We can retain slower variables by ignoring faster variables. Confirmation of the order parameter can help us further understand a system's self-organization mechanism. According to the earlier analysis of the state variables, responsiveness is the order parameter. Therefore, we can obtain $q_{2}=2 \alpha \theta q_{1} / \beta$; $q_{3}=2 \alpha \theta q_{1} /\left(\theta \gamma-\alpha \gamma q_{1}\right)$ from simultaneous equations $d q_{2} / d t=0$ and $d q_{3} / d t=0$.

In addition, we can obtain a system's evolution equation for the order parameter $q_{1}$ by putting $q_{3}$ into $d q_{1} / d t$.

$$
\frac{d q_{1}}{d t}=\alpha \theta q_{1}-2 \alpha \gamma \theta^{2} q_{1} /\left(\theta \gamma-\alpha \gamma q_{1}\right)-2 \alpha^{2} \gamma \theta q_{1}^{2} /\left(\theta \gamma-\alpha \gamma q_{1}\right)+8 \alpha^{3} \theta^{2} q_{1}^{2} /\left(\theta \gamma-\alpha \gamma q_{1}\right)^{2}
$$

Based on $q_{2}=2 \alpha \theta q_{1} / \beta$, there will be a linear relationship between a system's profitability and its responsiveness. A system diverges when it meets the requirements of the threshold value. At that point, it faces a range of options to find control variable $\theta$ so as to gradually increase its profitability. Figure 1 indicates the change features of the order parameter around the threshold value. Responsiveness will increase as control variable $\theta$ increases and moves toward balance. In the new round of investment decisions, responsiveness will significantly improve when $\theta$ reaches the threshold value. Figure 2 shows the evolution track of profitability $d q_{3} / d q_{1}=2 \alpha \gamma \theta /\left(\theta \gamma-\alpha \gamma q_{1}\right)^{2}>0$ and indicates that structural complexity $q_{3}$ will constantly expand with the increase in responsiveness $q_{1}$. However, considering the risk associated with practical investment and that there is a limit to a system's development, a system's control decision will become crucial. Its evolution trail will be similar to that in Figure 2. 


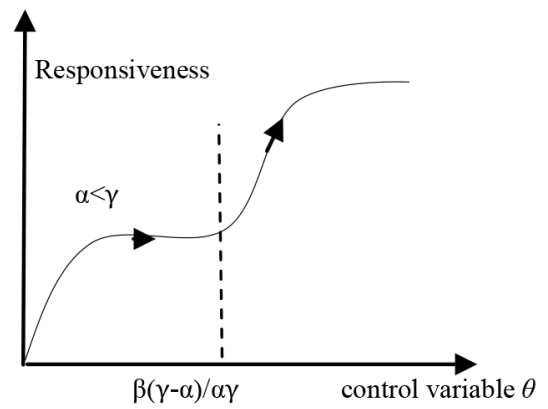

Figure 1. Change figure of system order parameter around threshold parameter when $\alpha<\gamma$

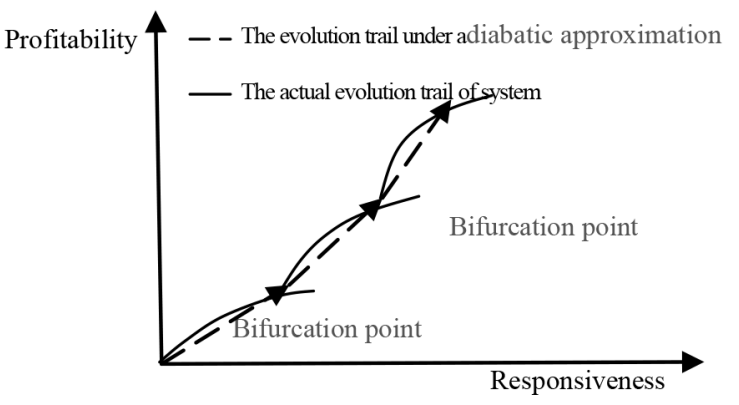

Figure 2. Evolution trail of profitability over responsiveness

\section{Simulation Example}

Considering the short life cycle of electronic products and various market demands, we choose an enterprise that manufactures electronic products in China, based on the average value of relevant data in the fourth quarter of $20 \mathrm{xx}$. To calculate $\alpha, \beta$, and $\gamma$, the standard value must be compared to dimensionless processing on the index. For this research, the standard value is regarded as the executive standard of the main manufacturing system. Based on that, we choose an additional three leading manufacturing enterprises and take the average value of indexes of the four as the executive standard.

\begin{tabular}{|c|r|r|r|r|r|}
\hline & \multicolumn{1}{|c|}{ A } & \multicolumn{1}{c|}{ B } & C & D & Standard value \\
\hline Sales margins (\%) & 5 & 4.5 & 6.1 & 4.8 & 5.1 \\
\hline Market share (\%) & 10.1 & 23.8 & 7.9 & 6.2 & 12 \\
\hline Ordering lead time (day) & 10 & 8 & 7 & 10 & 8.75 \\
\hline Order fill rate (\%) & 94 & 95 & 98 & 96 & 95.75 \\
\hline Quantity of new products promotion & 29 & 55 & 21 & 93 & 49.5 \\
\hline
\end{tabular}

Table 1. Statistical data of relevant indicators

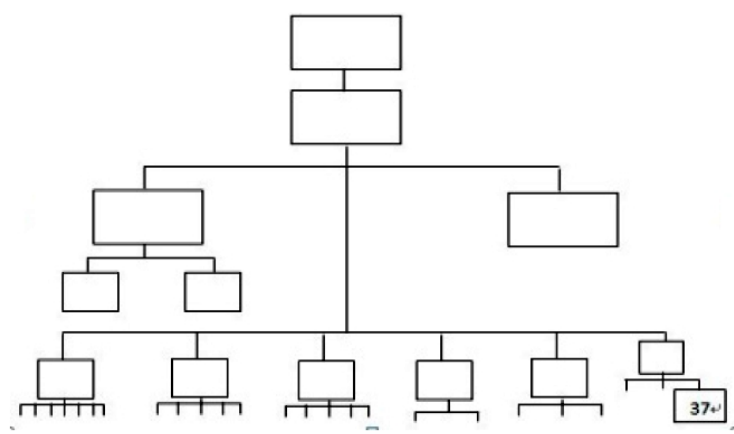

Figure 3. Organization chart of A enterprise 
Figure 3 indicates that the manufacturing enterprise system adopts a functional vertical structure -a U-shape structure- centralizing all the power in the top layer. "Management span" relates to the number of subordinate departments under direct management, while "decision path" relates to the shortest path the information can take from the first layer to the department. The directly connected length is 1 . The length will add 1 with each additional transfer. "Information communication chain" relates to the means of communication adopted by the internal management in each organization, for example, star chain 5, Y shape chain 3, and linear type chain 1 . Calculated by the author with the above dates, we can gain the results as shown in Table 2.

Combining the relevant data in Table 1 , we can determine the adjustment coefficient as $\alpha=0.2639, \beta=0.4542, \gamma=0.3074$. When a system faces a new strategic investment decision, using formula $\theta$ we can achieve healthy development by rationally distributing the level of investment in manpower, capital, and technology and ensure that a system meets the requirements of the threshold value. According to the analysis in section 4.1, the requirements of a system's threshold value are: $\theta=\beta(\gamma-\alpha) / \alpha \gamma=0.2436$. Simulation with Matlab software helps to obtain the evolution track of the order parameter when the control variable $\theta<0.2436$, $\theta>0.2436$ and $\theta=0.2436$, as shown in Figure 4.

\begin{tabular}{|c|c|c|c|c|c|}
\hline & $n$ & $\boldsymbol{S}_{\boldsymbol{i}}$ & $S_{m 2}$ & $y_{i}$ & $\overline{\gamma_{l}}$ \\
\hline Management span entropy $(i=1)$ & 36 & 2.9371 & 5.1699 & 0.4319 & 0.4672 \\
\hline Decision path entropy $(i=2)$ & 187 & 6.4860 & 7.5469 & 0.1406 & 0.1620 \\
\hline Information communication entropy $(i=3)$ & 72 & 4.6552 & 6.1699 & 0.2455 & 0.2521 \\
\hline
\end{tabular}

Table 2. Calculation results

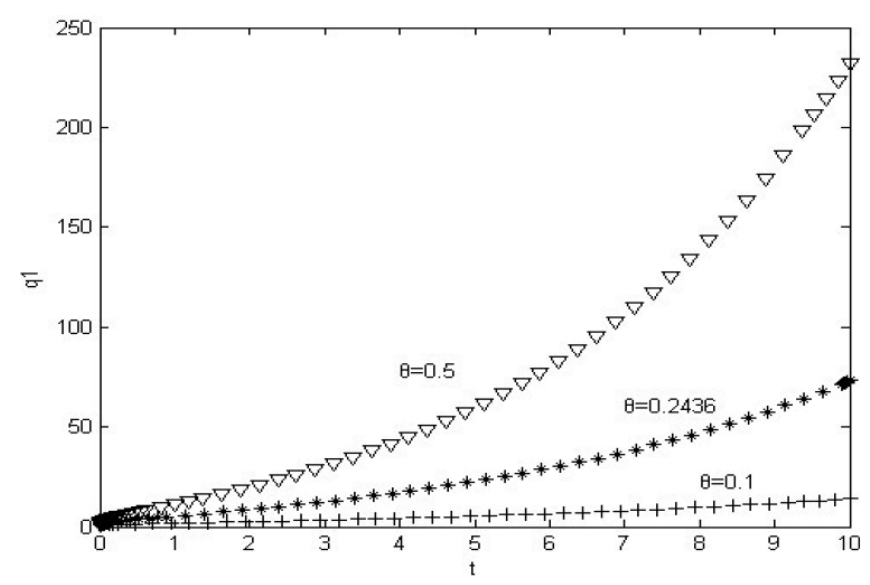

Figure 4. Evolution track of order parameter 
Figure 4 indicates that: (i) $\theta=0.1$ means a reduced external investment level. Under this condition, a system may be able to realize ordered evolution as time goes by. However, if the level of the order parameter remains low for an extended period, the level of the control variable will make it difficult to realize the goal of a manufacturing system responding rapidly to market demands. (ii) $\theta=0.2436$ simulates the evolution trail of the order parameter by changing the control variable to reach the level of the threshold value. At that point, the order parameter grows from nothing, and develops steadily toward a high ordered state. (iii) $\theta=0.5$ means controlling the external investment level above the threshold value level. At that point, the order parameter exists and constantly increases in level. As the control variable continues to increase in level, the evolution speed of the order parameter becomes relatively faster, which helps it respond to the market more swiftly and more appropriately. However, this does not mean that an unlimited increase in investment is necessary; factors such as the market, policy and strategy should be taken into account.

To sum up, when $\theta=0.2436$, the evolving curve is a system's threshold line of balance. Above the curve, a system can rapidly realize self-organization co-evolution. Under the curve, a system will be unstable for a period of time. The evolution level of the order parameter will remain low even if it can realize ordered evolution. To achieve orderly evolution and sustained profitability, a system assesses the best way to take full advantage of existing resources, makes a concerted effort to bring its evolution curve toward the upward side, and makes use of the threshold value condition to make investment decisions for new strategic investments.

\section{Conclusion and Future Work}

From the perspective of a complex system, this paper studies the co-evolution mechanism and stability analysis of an SOM enterprise system. Synergetics provides an appropriate method to describe the mechanism of action between the state variables. To find the relationship between system evolution and order parameter, we apply Lyapounov's indirect method and adiabatic approximation to analyze the evolution mechanism and stability, and lastly confirm the threshold condition of system's development.

The results show that the order parameter responsiveness has a long-term effect on an SOM enterprise system's operation, and a reasonable external investment level can improve effectively system's response capability and guide the system evolution from the current structure to a high ordered structure. In contrast, an unreasonable decision about investment level may not only make system be unstable but also have an inconspicuous rise in responsiveness. Gaining the system's threshold line of balance is crucial for a SOM enterprise system's development. Although it is difficult for decision makers to monitor an SOM enterprise system according to the threshold condition in the actual management process, it is necessary 
to achieve and maintain this condition by any means to realize the goal of resource collaboration and positive progress.

It should be noted that, in the example above, the SOM enterprise system is a single manufacturing enterprise, and as mentioned before, an SOM system can also be a cooperative alliance composed of mutually advantageous enterprises in the manufacturing supply chain, in which the manufacturing enterprise is the core. In this situation, an SOM system's evolution mechanism and threshold condition may be different in terms of the selection of variables and the measurement of indices. The cooperation mechanism and the model of a rapidly responsive supply chain thus merit further study. Moreover, this research shows the need for a system to indirectly form a monitoring index system.

\section{Acknowledgement}

This work is partly supported by Natural Science Foundation of China under grant NO. 71571072,71071057 and 50675069.

\section{References}

Basole, R.C., \& Bodner, D.A. (2015). Computational modeling of complex enterprise systems: a multi-level approach. Modeling and simulation in the systems engineering life cycle (Chapter 28). London: Springer-Verlag. http://dx.doi.org/10.1007/978-1-4471-5634-5_28

Fry, T.D., Steele, D.C., \& Saladin, B.A. (1994). A service-oriented manufacturing strategy. International Journal of Operations \& Production Management, 14(10), 17-29. http://dx.doi.org/10.1108/01443579410067225

Franco, R.D., Bas, Á.O., \& Esteban, F. (2009). Modeling extended manufacturing process with service-oriented entities. Service Business, 3(1), 31-50. http://dx.doi.org/10.1007/s11628-008-00560

Greenfield, H.I. (1996). Manpower and the growth of producer services. New York: Columbia University Press.

Gunasekaran, A., \& Spalanzani, A.(2012). Sustainability of manufacturing and services: investigations for research and applications. International Journal of Production Economics, 137(1), 116-125. http://dx.doi.org/10.1016/j.ijpe.2011.05.011

Grönroos, C. (2000). Service Management and Marketing: A Customer Relationship Management Approach. New Jersey: John Wiley \& Sons. 
Huang, S.X., Zeng, S., Fan, Y.S., \& Huang, G.Q. (2011). Optimal service selection and composition for service-oriented manufacturing network. International Journal of Computer Integrated Manufacturing, 24(5), 416-430. http://dx.doi.org/10.1080/0951192X.2010.511657

Lightfoot, H., Baines, T., \& Smart, T. (2013). The servitization of manufacturing: A systematic literature review of interdependent trends. International Journal of Operations \& Production Management, 33(11/12), 1408-1434. http://dx.doi.org/10.1108/17410380910960984

Macpherson, A. (1997). The role of producer service outsourcing in the innovation performance of New York State manufacturing firms. Annals of the Association of American Geographers, 87(1), 52-71. http://dx.doi.org/10.1111/0004-5608.00041

Mendes, J.M., Leitao, P., Colombo, A.W., \& Restivo, F. (2012). High-level Petri nets for the process description and control in service-oriented manufacturing systems. International Journal of Production Research, 50(6), 1650-1665. http://dx.doi.org/10.1080/00207543.2011.575892

Nariaki, N., \& Wang, S.H. (2013). Five models of platform-type product service systems in manufacturing. Procedia CIRP, 7, 389-394. http://dx.doi.org/10.1016/j.procir.2013.06.004

Paulo, L., Jose, B., \& Damien, T. (2012). Bio-inspired multi-agent systems for reconfigurable manufacturing systems. Journal of Engineering Applications of Artificial Intelligence, 25(5), 934-944. http://dx.doi.org/10.1016/j.engappai.2011.09.025

Qu, K., \& Ortoleva, P. (2007). Understanding stem cell differentiation through self-organization theory. Journal of Theoretical Biology, 250(4), 606-620. http://dx.doi.org/10.1016/j.jtbi.2007.10.019

Schmenner, R.W. (2004). Service businesses and productivity. Decision Science, 35(3), 333-347. http://dx.doi.org/10.1111/j.0011-7315.2004.02558.x

Schmenner, R.W. (2009). Manufacturing, service, and their integration: some history and theory. International Journal of Operations \& Production Management, 29(5), 431-443. http://dx.doi.org/10.1108/01443570910953577

Shin, M., Mun, J., \& Jung, M. (2009). Self-evolution framework of manufacturing systems based on fractal organization. Journal of Computers and Industrial Engineering, 56(3), 1029-1039. http://dx.doi.org/10.1016/j.cie.2008.09.014

Sarimveis, H., Patrinos, P., Tarantilis, C.D., \& Kiranoudis, C.T. (2008). Dynamic model and control of supply chain system: a review. Computers \& Operations Research, 35(11), 3530-3561. http://dx.doi.org/10.1016/j.cor.2007.01.017

Tharumarajah, A. (2003). A self-organizing view of manufacturing enterprise. Computers in Industry, 51(2), 185-186. http://dx.doi.org/10.1016/S0166-3615 (03)00035-6 
Tschacher, W., \& Haken, H. (2007). Intentionality in non-equilibrium systems? The functional aspects of self-organized pattern formation. New Ideas in Psychology, 25(1), 1-15. http://dx.doi.org/10.1016/j.newideapsych.2006.09.002

Wu, B., Xi, L.F., \& Zhou, B.H. (2008). Service-oriented communication architecture for automated manufacturing system integration. International Journal of Computer Integrated Manufacturing, 21(5), 599-615. http://dx.doi.org/10.1080/09511920701370746

Zhen, L. (2012). An analytical study on service-oriented manufacturing strategies. International Journal of Production Economics, 139(1), 220-228.

http://dx.doi.org/10.1016/j.ijpe.2012.04.010

Journal of Industrial Engineering and Management, 2015 (www.jiem.org)

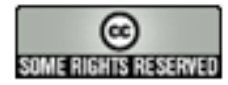

Article's contents are provided on an Attribution-Non Commercial 3.0 Creative commons license. Readers are allowed to copy, distribute and communicate article's contents, provided the author's and Journal of Industrial Engineering and Management's names are included. It must not be used for commercial purposes. To see the complete license contents, please visit http://creativecommons.org/licenses/by-nc/3.0/. 